\title{
Entrenamiento de fuerza y resistencia en hipoxia: efecto en la hipertrofia muscular
}

\author{
Diego Fernández-Lázaro ${ }^{1}$, Joseba Díaz² ${ }^{2}$ Alberto Caballero ${ }^{3}$, Alfredo Córdova $^{4}$ \\ ${ }^{1}$ Departamento de Biología Celular, Histología y Farmacología, Facultad de Fisioterapia, \\ Universidad de Valladolid, Soria, España \\ ${ }^{2}$ Centro Deportivo ElikaSport, Gipuzkoa, España \\ ${ }^{3}$ Departamento de Fisiología, Facultad de Fisioterapia, Universidad de Valladolid, Soria, España \\ ${ }^{4}$ Departamento de Anatomía, Facultad de Fisioterapia, Universidad de Valladolid, Soria, España
}

El entrenamiento en altitud y el entrenamiento en hipoxia simulada producen adaptaciones fisiológicas y bioquímicas en el músculo esquelético como la capacidad oxidativa, así como modificaciones de la actividad mitocondrial, en el metabolismo aerobio y en el contenido de mioglobina.

El propósito de esta revisión fue analizar las adaptaciones del músculo esquelético en respuesta a la exposición temporal a la hipoxia combinada con ejercicios de fuerza y resistencia. Según los hallazgos de numerosos autores, las adaptaciones estructurales del músculo son similares en la hipoxia y en la 'normoxia', con excepción de un aumento en el volumen muscular y en el área de la sección transversal de la fibra muscular, que son mayores en la hipoxia.

En conclusión, la sinergia del entrenamiento de fuerza y resistencia y la hipoxia normobárica produce mejores y mayores adaptaciones, ganancias y cambios fisiológicos beneficiosos en el tejido muscular, lo cual genera cambios fenotípicos favorables, como la hipertrofia del músculo esquelético.

Palabras clave: hipoxia; ejercicio; músculo; hipertrofia; fuerza muscular; entrenamiento de resistencia.

\section{The training of strength-resistance in hypoxia: effect on muscle hypertrophy}

Altitude and simulated-hypoxia training produces different physiological and/or biochemical adaptations in the skeletal muscle. These are: oxidative capacity, mitochondrial activity modifications, aerobic metabolism changes and myoglobin content.

The purpose of this review was to analyze the adaptations of skeletal muscle in response to the combination of strength-resistance exercise and hypoxia. In general terms, the structural adaptations of the muscle are similar in hypoxia and normoxia except that hypoxia training produces an increase of the volume and cross-sectional area of the muscle fiber.

Citación:

Fernández-Lázaro D, Díaz J, Caballero A, Córdova A. Entrenamiento de fuerza y resistencia en hipoxia: efecto en la hipertrofia muscular. Biomédica. 2019;39:212-20

https://doi.org/10.7705/biomedica.v39i2.4084

Correspondencia:

Alfredo Córdova, Facultad de Fisioterapia,

Universidad de Valladolid, Campus Universitario de

Soria, 42003 Soria, España

a.cordova@bio.uva.es

Contribución de los autores:

Diego Fernández-Lázaro: participación en todas las etapas del estudio

Alfredo Córdova: concepción y diseño del estudio, análisis e interpretación de los datos

Alberto Caballero y Joseba Díaz: recolección de los datos

Todos los autores participaron en la escritura del manuscrito.

Financiación:

No se requirió financiación.

Conflicto de intereses:

Ninguno In conclusion, the synergic effect of the combination of strength resistance training with normobaric hypoxia produces better and greater adaptations and beneficial physiological changes of the muscle tissue, which shows favorable phenotypic changes in skeletal muscle hypertrophy.

Key words: Hypoxia; exercise; muscle; hypertrophy; muscle strength; resistance training.

La hipoxia como método de entrenamiento adquirió mayor importancia tras las olimpiadas celebradas en la ciudad de México $(2.300 \mathrm{~m})$ en 1968. Numerosas investigaciones han evidenciado los beneficios de 'entrenar arriba y vivir abajo', con el fin de aprovechar mejor los efectos del entrenamiento en altitud (1). El entrenamiento en altitud y el entrenamiento en hipoxia simulada producen distintas adaptaciones fisiológicas y bioquímicas en el músculo esquelético $(2,3)$.

Además, el ejercicio de fuerza y resistencia produce por sí mismo una serie de adaptaciones morfológicas en el tejido muscular esquelético (4), especialmente cambios en la hipertrofia, la fuerza, el diámetro de la fibra, la síntesis de miofibrillas y un aumento de la capacidad anaerobia. Otras adaptaciones menos notorias observadas en el músculo esquelético son la síntesis mitocondrial y la tolerancia al lactato, así como el mejoramiento de la función oxidativa y de la capacidad de resistencia (4). 
El propósito de la presente revisión fue describir las adaptaciones hipertróficas del músculo esquelético en respuesta a la exposición temporal a situaciones de hipoxia combinada con ejercicios de fuerza y resistencia.

\section{Metodología}

Se hizo una búsqueda bibliográfica entre marzo y julio de 2017 en la base de datos Pubmed de la U.S. National Library of Medicine, sin restricción de fechas de publicación o idioma,

La búsqueda se centró en tres tipos de estudios (cuadro 1):

1) estudios sobre el uso de la hipoxia y el rendimiento muscular con la combinación de los términos "hipoxia and performance" de los Medical Subject Headings (MeSH);

2) estudios sobre el entrenamiento en hipoxia y el músculo esquelético, con la combinación de términos MeSH "hipoxia training and skeletal muscle", y

3) estudios sobre la hipoxia intermitente y el músculo combinando los términos MeSH "intermittent hypoxia and muscle".

Cuadro 1. Relación de términos de búsqueda y publicaciones encontradas

\begin{tabular}{|c|c|c|}
\hline \multicolumn{2}{|c|}{ Término de búsqueda } & \multirow{2}{*}{$\begin{array}{c}\text { Número de } \\
\text { publicaciones }\end{array}$} \\
\hline Inglés & Español & \\
\hline $\begin{array}{l}\text { Hypoxia AND muscle } \\
\text { performance }\end{array}$ & $\begin{array}{l}\text { Hipoxia AND rendimiento } \\
\text { muscular }\end{array}$ & 82 \\
\hline $\begin{array}{l}\text { Hypoxia training AND } \\
\text { muscle }\end{array}$ & $\begin{array}{l}\text { Entrenamiento en hipoxia } \\
\text { AND músculo }\end{array}$ & 112 \\
\hline $\begin{array}{l}\text { Intermittent hipoxia AND } \\
\text { muscle }\end{array}$ & $\begin{array}{l}\text { Hipoxia intermitente AND } \\
\text { músculo }\end{array}$ & 47 \\
\hline
\end{tabular}

Además, se emplearon dos tesis doctorales, una dirigida por uno de los autores de la revisión, que contenía como información relevante el empleo de la hipoxia intermitente en atletas de élite (3), y una segunda tesis con información actualizada sobre enfermedades crónicas (5). Se incluyó, asimismo, un libro que explicaba las claves fisiológicas del entrenamiento en altitud (6).

\section{Resultados}

A partir del número total de publicaciones encontradas con los términos de búsqueda (cuadro 2), se seleccionaron 37 tras la aplicación de los criterios de inclusión: artículos relacionados con el entrenamiento en hipoxia, estudios específicos sobre el músculo esquelético y sobre la actividad física o deportiva, y de los criterios de exclusión: artículos relacionados con enfermedades que no fueran musculares y estudios que no se referían al músculo. Estos criterios se emplearon para analizar los documentos más relevantes por su pertinencia temática dada la abundancia de manuscritos que incluyen los términos hipoxia y músculo.

Cuadro 2. Efectos del entrenamiento físico

\begin{tabular}{ll}
\hline Cardiacos & Contra la arritmia, de regulación de la presión sanguínea \\
\hline Hematológicos & Mejoras hemodinámicas, antitrombóticos \\
Metabólicos & Perfil lipídico: $L L D L$ y $\uparrow \mathrm{HDL}$ \\
Inmunológicos & Rerfil glucémico: $\uparrow$ sensibilidad insulina y łaprovechamiento de la glucosa \\
\hline
\end{tabular}

LDL: lipoproteinas de baja densidad (low-density lipoprotein); HDL: lipoproteinas de alta densidad (highdensity lipoproteins) 


\section{Hipoxia}

La hipoxia se define como la reducción del contenido o de la presión parcial de oxígeno $\left(\mathrm{O}_{2}\right)$ a nivel celular. Se conocen varios tipos de hipoxia que responden a las causas que la provocan y al tiempo de exposición. Desde el punto de vista de las causas, se han descrito la hipoxia anémica, la hipoxia por estancamiento, la citotóxica y la hipoxémica. Esta última es la más relevante para el mejoramiento del rendimiento muscular (7) e incluye dos subtipos: la hipoxia hipobárica con presión atmosférica menor a 760 $\mathrm{mm} \mathrm{Hg}$ y una concentración de $\mathrm{O}_{2}$ en el aire $\left(\mathrm{FiO}_{2}\right)$ de 20,9\%, y la hipoxia normobárica, con una presión barométrica de $760 \mathrm{~mm} \mathrm{Hg}$ y una $\mathrm{FiO}_{2}$ menor de $20,9 \%$. Se pueden generar condiciones de hipoxia artificial hipobárica o normobárica mediante dispositivos como las máscaras con mezclas de gases, las cámaras normobáricas, las tiendas o habitaciones de hipoxia y los dispositivos respiratorios empobrecidos en oxígeno (8).

En cuanto al tiempo de exposición, la hipoxia se clasifica en aguda, crónica o intermitente. La aguda corresponde a una exposición breve; la crónica, a periodos prolongados, incluso años, y, generalmente, compromete a las personas nacidas en altitud o que residen permanentemente en lugares altos, en tanto que la intermitente es aquella en que los sujetos se someten a ciclos alternados de hipoxia y 'normoxia'; actualmente esta es la más empleada para mejorar el rendimiento deportivo (9).

Para el control de la hipoxia, la medida de la saturación arterial de oxígeno $\left(\mathrm{SaO}_{2}\right)$ como parámetro para regular la intensidad el estímulo hipóxico sobre el organismo es más efectiva, estable e individualizada que la utilización de la $\mathrm{FiO}_{2}$ o el nivel de hipoxia (10). Para producir una respuesta de estrés significativa, la $\mathrm{SaO}_{2}$ debe ser inferior al $90 \%$ (11).

\section{Efectos fisiológicos del entrenamiento}

Cardiometabólicos. Un estilo de vida saludable exige la práctica de la actividad física y del ejercicio como aspecto fundamental para la prevención, el manejo y el tratamiento de muchas enfermedades crónicas, entre ellas las cardiometabólicas (12). Algunas de estas enfermedades, como la hipertensión, las cardiopatías, la obesidad y la diabetes mellitus tipo 2, son las principales causas de mortalidad a nivel mundial (5). Son múltiples los efectos protectores del ejercicio contra la arritmia (aumento de tono vagal, disminución de la actividad adrenérgica, regulación del ritmo cardiaco) y contra la trombosis (aumento de la fibrinólisis, disminución de la agregación plaquetaria, menor viscosidad de la sangre), así como de mejoría hemodinámica (aumento del flujo coronario, remodelación del músculo cardiaco, mejora de la función del endotelio vascular, aumento de la producción de óxido nítrico con efecto vasodilatador, y un eficiente aprovechamiento del oxígeno por parte del miocardio).

Por otra parte, las adaptaciones metabólicas como el aumento de la sensibilidad a la insulina se traducen en un mejor aprovechamiento de la glucosa (12), en la disminución de las lipoproteínas de alta densidad (highdensity lipoprotein, HDL), el aumento de las de baja densidad (low-density lipoprotein, LDL), la disminución de los triglicéridos y la adiposidad, la regulación de las citocinas responsables de los procesos inflamatorios, y la regulación de los mecanismos de la presión sanguínea, fenómenos que contribuyen a la homeostasis y, por lo tanto, a un mejor funcionamiento de otros órganos y sistemas. 
Musculares (hipertrofia muscular). La práctica habitual de ejercicio físico produce aumentos en la masa del músculo esquelético debidos a la hipertrofia inducida por los mecanismos que se presentan en la figura 1. El proceso de hipertrofia de la fibra del músculo esquelético surge como resultado de la confluencia del equilibrio de las proteínas musculares y la adición de células satélites a las fibras musculares esqueléticas. El equilibrio positivo de la proteína muscular se logra cuando la tasa de la nueva síntesis de proteínas musculares supera el de su descomposición (13) y de la actuación de las células satélites musculares encargadas de la remodelación y la hipertrofia del tejido muscular (14).
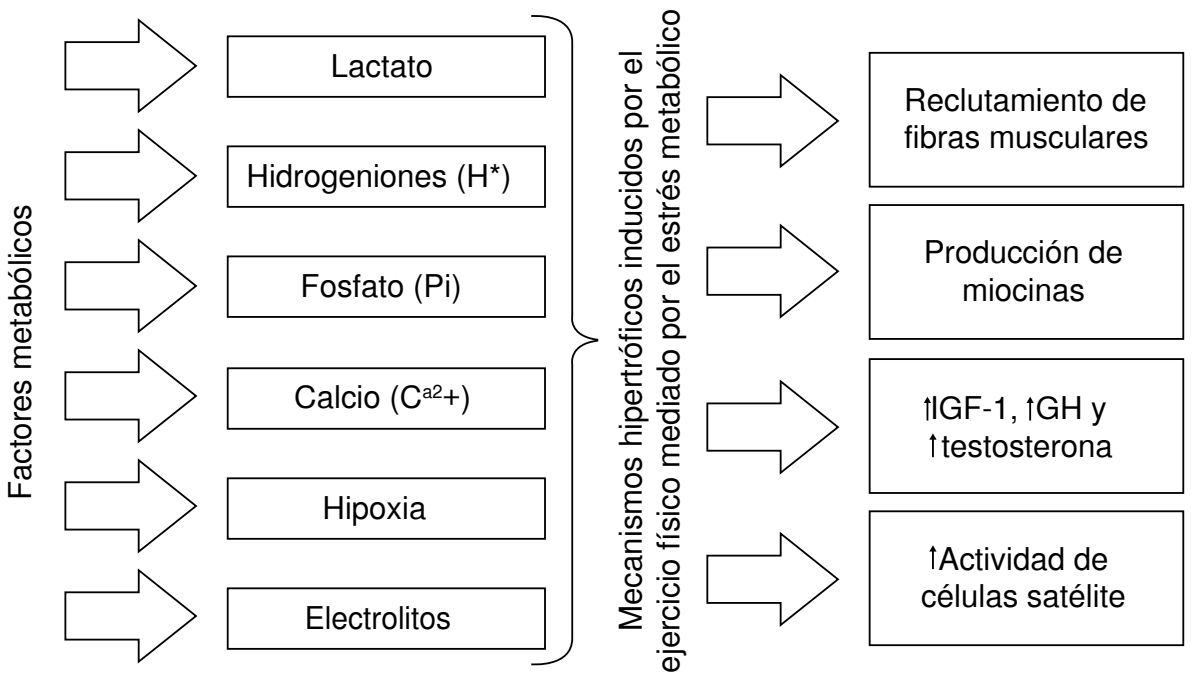

Figura 1. Factores metabólicos involucrados en la hipertrofia muscular IGF-1: factor de crecimiento insulínico tipo 1; GH: hormona del crecimiento

\section{Condicionantes fisiopatológicos de la hipoxia}

Es necesario conocer y considerar los riesgos de las condiciones de hipoxia. En dos de los estudios consultados $(2,15)$, se describen algunas de las repercusiones nocivas, como la fatiga con alteraciones neuromusculares, el aumento del estrés oxidativo, las alteraciones inmunológicas e inflamatorias, los efectos sobre el árbol vascular (vasoconstricción), la excesiva policitemia, los trastornos del ritmo ventricular y supraventicular, las perturbaciones psicológicas, la ansiedad, la depresión, las lagunas cognitivas y la dificultad para expresar emociones.

El conocimiento de tales efectos permite adecuar más fácilmente las condiciones de la hipoxia y de su empleo conjunto con la actividad física en el entrenamiento deportivo para conseguir las adaptaciones musculares deseadas.

\section{Adaptaciones y respuestas musculares inducidas por el entrenamiento en hipoxia}

El uso adecuado de la hipoxia normobárica o hipobárica durante el ejercicio incrementa las funciones metabólicas del tejido muscular esquelético (16). El músculo posee gran plasticidad, lo que le confiere la capacidad de efectuar su función en todo tipo de circunstancias de la manera más eficiente. Las adaptaciones a la hipoxia modifican cualidades bioquímicas, como la capacidad oxidativa y la actividad mitocondrial, y producen cambios en el metabolismo aerobio y en el contenido de mioglobina (11) en la célula muscular esquelética. 
Las condiciones hipóxicas en el tejido muscular inducen eventos de señalización específicos que provocan cambios en el fenotipo muscular por la activación de los llamados factores inducibles por hipoxia (hipoxia inducible factor, HIF) y la señalización mediada por estos afecta la expresión de un gran número de genes (17), muchos de los cuales tienen un significado funcional en el tejido del músculo esquelético (18). Además, los HIF son la clave para la expresión de los genes relacionados con la eritropoyesis y la angiogénesis, y de aquellos relacionados con la regulación del pH y la glucólisis (19).

Por otra parte, se ha reportado que las adaptaciones estructurales musculares son similares en la hipoxia y en la 'normoxia', con excepción de un aumento en el volumen muscular y en el área de la sección transversal de la fibra muscular, lo que se consigue con el entrenamiento en hipoxia (20). Los mecanismos responsables de estas trasformaciones se deben también a la acción de los HIF, la cual estimula los genes reguladores de la hipertrofia muscular y, además, promueve un incremento de los niveles de respuesta hormonal anabólica, como el del factor de crecimiento insulinoide de tipo 1 (Insulin-Like Growth Factor-1, IGF-1), de la testosterona y de la hormona del crecimiento (21-26). Por lo tanto, el entrenamiento en hipoxia, comparado con el entrenamiento en 'normoxia', tiene efectos sinérgicos y diferenciales sobre el tejido muscular esquelético (27).

\section{La hipertrofia muscular por el entrenamiento en hipoxia}

Feriche, et al., evidenciaron en su estudio los cambios hipertróficos musculares debidos a la acumulación de metabolitos (28) (figura 1) mediante entrenamientos con seis a 12 repeticiones, e intensidades mayores al $65 \%$ de una repetición máxima. Otro tipo de entrenamiento con el cual se consiguieron los mismos cambios fenotípicos consistió en la realización de cinco bloques de 15 repeticiones de ejercicios de baja resistencia ( $\leq 30 \%$ de una repetición máxima), con una recuperación de 90 segundos, lo que favoreció el aumento de repeticiones con menores periodos de descanso, rutina que se prolongó durante cinco semanas. Según estos autores (28), se consiguen mayores cambios hipertróficos musculares mediante el empleo de dispositivos que consigan crear unas condiciones de hipoxia normobárica, con altitudes simuladas de 2.500 a $3.000 \mathrm{~m}$, en las cuales la $\mathrm{FiO}_{2}$ es 13 a $16 \%$ mayor que en condiciones de 'normoxia'.

Por su parte, Nishimura, et al., sugieren en su estudio que el entrenamiento de resistencia bajo condiciones de hipoxia mejora la fuerza muscular e induce la hipertrofia muscular de manera significativa y con mayor rapidez que bajo condiciones de 'normoxia' (26). Los autores lo consiguieron con períodos de entrenamiento de seis semanas al $70 \%$ de una repetición máxima en series de diez repeticiones y en condiciones de hipoxia normobárica ( $16 \%$ de $\mathrm{FiO} 2$ ) en comparación con las condiciones de 'normoxia' (26). El resultado fue un aumento entre 1,3 y 1,9\% en el área de la sección transversal de la fibra muscular.

En un estudio del 2015, Kurobe, et al. (23) dividieron a los participantes en dos grupos. Uno de ellos se ejercitó en condiciones de 'normoxia' y el otro en hipoxia normobárica ( $12,7 \%$ de $\mathrm{FiO}_{2}$ ) durante 95 minutos. El protocolo de entrenamiento de los dos grupos consistió en repeticiones de extensión de hombro con 10 repeticiones máximas en intervalos de máximo un minuto, tres veces a la semana durante un periodo total de ocho semanas. Se comprobó un aumento mayor de los niveles de la hormona del crecimiento en el grupo entrenado en hipoxia en comparación con el entrenado en 'normoxia'. A pesar de este aumento de 
la hormona, no hubo ningún cambio estructural en el músculo esquelético, pero sí en la funcionalidad muscular, debido a que los sujetos estimulados con hipoxia fueron capaces de un mayor número de repeticiones. Estos resultados concuerdan con los del estudio de Schroeder, et al. (29), quienes señalaron que la elevación de los niveles de las hormonas anabólicas, como la del crecimiento, no serían necesarias para estimular la síntesis de proteínas musculares o la hipertrofia de pequeñas masas musculares, aunque son ideales para optimizar el rendimiento funcional de la masa muscular entrenada.

Bajo condiciones simuladas de hipoxia normobárica hasta conseguir una $\mathrm{SaO}_{2}$ del $80 \%$ con el empleo de máscaras hipóxicas, Manimmanakorn, et al., observaron un incremento del 3,2 \% del área de la sección transversal de la fibra muscular tras un entrenamiento de cinco semanas con cargas de baja resistencia ( $20 \%$ de una repetición máxima), consistentes en tres repeticiones como máximo y una recuperación de 30 segundos $(24,25)$.

Por su parte, Brocherie, et al., combinaron el ejercicio físico en condiciones de hipoxia normobárica con la circunstancia de vivir en la altitud, lo cual significó un doble estímulo hipóxico (30). El protocolo seguido por los deportistas consistió en vivir durante 14 días en hipoxia normobárica (más de $14 \mathrm{~h}$ al día con una $\mathrm{FiO}_{2}$ entre 14,5 y 14,2\%). Cada una de las seis sesiones realizadas incluía cinco bloques de cinco segundos de un ejercicio de esprint de máxima intensidad con una recuperación pasiva de 25 segundos entre cada ejercicio y cinco minutos entre bloques bajo una hipoxia normobárica correspondiente a la de una altitud simulada de $3.000 \mathrm{~m}$ y una $\mathrm{FiO}_{2}$ de $15 \mathrm{a}$ $12 \%$. En el estudio se reportó un aumento de los niveles de HIF-1a, lo que se tradujo en un aumento del factor de crecimiento vascular (Vascular Endothelial Growth Factor, VEGF) y de los niveles de mioglobina. Además, se observó una proliferación de células musculares, muy probablemente debida al incremento del factor de crecimiento insulinoide 2 (IGF-2) estimulado por el HIF-1a (7).

Por otra parte, Friedmann, et al., observaron un aumento de la resistencia muscular en individuos que realizaron durante cuatro semanas una rutina de ejercicios bajo condiciones de hipoxia normobárica con una $\mathrm{FiO}_{2}$ del $12 \%$ comparado con los sujetos entrenados en 'normoxia' (21). El entrenamiento consistió en extensiones de rodilla $(6 \times 25)$ para trabajar los cuádriceps con $30 \%$ de repetición máxima y periodos de recuperación de 25 segundos. El aumento de la resistencia muscular se tradujo en un aumento del área de la sección transversal de la fibra muscular y del número de fibras musculares en el grupo de hipoxia normobárica comparado con el grupo de control.

En su estudio, Kon, et al., emplearon ejercicios de los músculos pectorales y prensa de piernas con $70 \%$ de una repetición máxima en un ambiente de hipoxia normobárica y una $\mathrm{FiO}_{2}$ del $14 \%$ y observaron que los efectos con respecto al grupo de control entrenado en 'normoxia' se tradujeron en un aumento del área de la sección transversal de la fibra muscular, un incremento significativo de la resistencia muscular, una mayor concentración plasmática del VEGF y una relación entre capilar y fibra del músculo esquelético significativamente mayor (22). En resumen, los autores registraron un aumento del tamaño, la fuerza y la resistencia musculares, así como de la angiogénesis del músculo esquelético.

\section{Rutinas del entrenamiento en hipoxia}

Otro punto clave para mejorar el rendimiento y conseguir la hipertrofia muscular es la rutina de ejercicios que se prescriba. Surge la pregunta de cómo el estímulo hipóxico puede desencadenar fenómenos de adaptaciones 
beneficiosas, como la hipertrofia del músculo esquelético, cuando también puede producir su degeneración y atrofia en condiciones de hipoxia extrema $(31,32)$.

En nuestra opinión, el estímulo hipóxico para maximizar los efectos positivos debe darse únicamente durante la sesión de entrenamiento, es decir, durante la contracción muscular, lo que permitiría al sujeto la recuperación muscular en 'normoxia'. Por lo tanto, la aplicación del paradigma de 'vivir abajo y entrenar arriba' supondría la inducción de adaptaciones musculares que mejoran el rendimiento del tejido muscular mediante un único estímulo hipóxico durante el entrenamiento.

Las sesiones de ejercicio en hipoxia suponen para el organismo un mayor desgaste, fatiga, inmunosopresión y estrés orgánico que las realizadas en 'normoxia' (32) y, por ende, los periodos de recuperación también deberían ser mayores (33). En consecuencia, en el entrenamiento en hipoxia deben considerarse las premisas anteriores para evitar los efectos negativos sobre el tejido muscular.

\section{Importancia de la dieta en el entrenamiento en hipoxia}

Cabe resaltar que en los estudios analizados no se consideraron los detalles de la dieta y los complementos alimentarios ingeridos por los participantes durante el periodo de estudio. Bajo condiciones de hipoxia, las demandas energéticas aumentan debido al mayor incremento de las hormonas simpático-adrenales $(2,3,27)$, por lo tanto, es necesario consumir una dieta adecuada durante las sesiones de entrenamiento en hipoxia (32).

Estos suplementos alimentarios deben garantizar la síntesis de proteínas musculares, ya que la adaptación muscular que se busca es la hipertrofia. Para que dicha síntesis sea adecuada, se necesita una dieta rica en proteínas que contengan aminoácidos esenciales, arginina y glutamina, así como el consumo de carbohidratos (13). Con estos sustratos se asegura la efectividad de la síntesis y la ganancia de masa del músculo esquelético (34). Por lo tanto, aunque en los individuos en hipoxia se desencadenen de manera más potente los mecanismos de incremento del tejido muscular, en ausencia de los sustratos necesarios, las ganancias de músculo se ven limitadas y podrían no ser significativas en comparación con la de los grupos en 'normoxia' e, incluso, supondrían un equilibrio negativo entre la síntesis y el catabolismo y, en consecuencia, crearían el efecto contrario (atrofia) del que se pretende (hipertrofia).

Otras recomendaciones nutricionales que no se mencionan en los artículos señalados y que deben tenerse en cuenta son la hidratación (35) y los suplementos minerales en la alimentación (36). En este sentido, Córdova, et al., demostraron que bajo condiciones hipóxicas se produce un descenso del magnesio en el corazón, el hígado, el riñón y, más importante incluso, en la musculatura esquelética (37), lo cual sugiere que los suplementos con magnesio cuando se hacen ejercicios en hipoxia pueden ser un elemento clave.

\section{Conclusiones}

Esta revisión permitió concluir que la sinergia del entrenamiento y la hipoxia normobárica produce mejores y mayores adaptaciones, ganancias y cambios fisiológicos beneficiosos en el tejido muscular, lo cual trae consigo transformaciones fenotípicas favorables como la hipertrofia del músculo esquelético cuando se garantizan condiciones óptimas de entrenamiento e hipoxia. 
Para ello deben considerarse la dosis de hipoxia, la duración de las sesiones, la intensidad del ejercicio, la frecuencia semanal, las semanas de tratamiento y el número de sesiones. Además, deben contemplarse los aspectos dietéticos y nutricionales, así como la administración de los nutrientes necesarios.

\section{Agradecimientos}

Al instituto de Estudios de Ciencias de la Salud de Castilla y León (ICSCYL) por su apoyo y colaboración en todo el proceso de elaboración del estudio.

\section{Referencias}

1. De Smet S, van Herpt P, D'Hulst G, Van Thienen R, Van Leemputte M, Hespel P. Physiological adaptations to hypoxic vs. normoxic training during intermittent living high. Front Physiol. 2017;8:347. https://doi.org/10.3389/fphys.2017.00347

2. Cordova-Martínez A, Pascual-Fernández J, Fernández-Lázaro D, Álvarez-Mon M. Muscular and heart adaptations of execise in hypoxia. Is training in slow hypoxy healthy? Med Clin (Barc). 2017;148:469-74. https://doi.org/10.1016/j.medcli.2017.02.013

3. Pascual-Oliva E. Efecto de la hipoxia intermitente en atletas de élite (tesis). Soria: Universidad de Valladolid; 2012.

4. Egan B, Zierath JR. Exercise metabolism and the molecular regulation of skeletal muscle adaptation. Cell Metab. 2013;17:162-84. https://doi.org/10.1016/j.cmet.2012.12.012

5. Fernández-Lázaro $\mathrm{Cl}$. Adherencia (sic) al tratamiento farmacológico en personas con patologías crónicas en grupos de población vulnerable (tesis). Salamanca: Universidad de Salamanca; 2017.

6. Urdampilleta A. Fisiologia de la hipoxia y entrenamientos en altitud. Primera edición. San Sebastian, España: Elikasport Publicaciones; 2015.

7. Lundby C, Calbet JA, Robach P. The response of human skeletal muscle tissue to hypoxia. Cell Mol Life Sci. 2009;66:3615-23. https://doi.org/10.1007/s00018-009-0146-8

8. Cabalet JA. Fisiologia de la altitud y entrenamiento físico. Fisiología del ejercicio. Primera edición. Buenos Aires: Editorial Panamericana; 2006.

9. Coppel J, Hennis P, Gilbert-Kawai E, Grocott MP. The physiological effects of hypobaric hypoxia versus normobaric hypoxia: A systematic review of crossover trials. Extrem Physiol Med. 2015;4:2. https://doi.org/10.1186/s13728-014-0021-6

10. Humphreys S, Deyermond R, Bali I, Stevenson M, Fee JP. The effect of high altitude commercial air travel on oxygen saturation. Anaesthesia. 2005;60:458-60. https://doi. org/10.1111/j.1365-2044.2005.04124.x

11. Millet GP, Roels B, Schmitt L, Woorons $X$, Richalet JP. Combining hypoxic methods for peak performance. Sports Med. 2010;40:1-25. https://doi.org/10.2165/11317920-000000000-00000

12. Kachur S, Chongthammakun V, Lavie CJ, De Schutter A, Arena R, Milani RV, et al. Impact of cardiac rehabilitation and exercise training programs in coronary heart disease. Prog Cardiovasc Dis. 2017;60:103-14.https://doi.org/10.1016/j.pcad.2017.07.002

13. Phillips SM. A brief review of critical processes in exercise-induced muscular hypertrophy. Sports Med. 2014;44:S71-7. https://doi.org/10.1007/s40279-014-0152-3

14. Farup J, Rahbek SK, Riis S, Vendelbo MH, Paoli F, Vissing K. Influence of exercise contraction mode and protein supplementation on human skeletal muscle satellite cell content and muscle fiber growth. J Appl Physiol (1985). 2014;117:898-909. https://doi.org/ 10.1152/japplphysiol.00261.2014

15. Caneva JO, Rabec CA, De Salvo MC, Mazzei JA. Physiopathology, diagnosis and treatment of severe chronic hypoxemia. Role of residential chronic oxygen therapy. Medicina (B Aires). 2001;61:453-69.

16. Hoppeler $\mathbf{H}$, Klossner $\mathbf{S}$, Vogt $\mathbf{M}$. Training in hypoxia and its effects on skeletal muscle tissue. Scand J Med Sci Sports. 2008;18:38-49. https://doi.org/10.1111/j.1600-0838.2008.00831.X

17. Semenza GL, Shimoda LA, Prabhakar NR. Regulation of gene expression by HIF-1. Novartis Found Symp. 2006;272:2-8.

18. Hoppeler H, Fluck M. Normal mammalian skeletal muscle and its phenotypic plasticity. J Exp Biol. 2002;205:2143-52. 
19. Lee JW, Bae SH, Jeong JW, Kim SH, Kim KW. Hypoxia-inducible factor (HIF-1)alpha: Its protein stability and biological functions. Exp Mol Med. 2004;36:1-12. https://doi. org/10.1038/emm.2004.1

20. Hoppeler H, Vogt M. Muscle tissue adaptations to hypoxia. J Exp Biol. 2001;204:3133-9.

21. Friedmann B, Kinscherf R, Borisch S, Richter G, Bartsch P, Billeter R. Effects of low-resistance/high-repetition strength training in hypoxia on muscle structure and gene expression. Pflugers Arch. 2003;446:742-51. https://doi.org/10.1007/s00424-003-1133-9

22. Kon M, Ohiwa N, Honda A, Matsubayashi T, Ikeda T, Akimoto T, et al. Effects of systemic hypoxia on human muscular adaptations to resistance exercise training. Physio Rep. 2014;2. https://doi.org/10.14814/phy2.12033

23. Kurobe K, Huang Z, Nishiwaki M, Yamamoto M, Kanehisa H, Ogita F. Effects of resistance training under hypoxic conditions on muscle hypertrophy and strength. Clin Physiol Funct Imaging. 2015;35:197-202. https://doi.org/10.1111/cpf.12147

24. Manimmanakorn A, Hamlin MJ, Ross JJ, Taylor R, Manimmanakorn N. Effects of low-load resistance training combined with blood flow restriction or hypoxia on muscle function and performance in netball athletes. J Sci Med Sport. 2013;16:337-42. https://doi. org/10.1016/j.jsams.2012.08.009

25. Manimmanakorn A, Manimmanakorn N, Taylor R, Draper N, Billaut F, Shearman JP, et al. Effects of resistance training combined with vascular occlusion or hypoxia on neuromuscular function in athletes. Eur J Appl Physiol. 2013;113:1767-74. https://doi.org/ 0.1007/s00421-013-2605-z

26. Nishimura A, Sugita M, Kato K, Fukuda A, Sudo A, Uchida A. Hypoxia increases muscle hypertrophy induced by resistance training. Int J Sports Physiol Perform. 2010;5:497-508. https://doi.org/10.1123/ijspp.5.4.497

27. McLean BD, Gore CJ, Kemp J. Application of 'live low-train high' for enhancing normoxic exercise performance in team sport athletes. Sports Med. 2014;44:1275-87. https://doi. org/10.1007/s40279-014-0204-8

28. Feriche B, García-Ramos A, Morales-Artacho AJ, Padial P. Resistance training using different hypoxic training strategies: A basis for hypertrophy and muscle power development. Sports Med Open. 2017;3:12. https://doi.org/10.1186/s40798-017-0078-Z

29. Schroeder ET, Villanueva M, West DD, Phillips SM. Are acute post-resistance exercise increases in testosterone, growth hormone, and IGF-1 necessary to stimulate skeletal muscle anabolism and hypertrophy? Med Sci Sports Exerc. 2013;45:2044-51. https://doi. org/10.1249/MSS.0000000000000147.

30. Brocherie F, Girard O, Faiss R, Millet GP. Effects of repeated-sprint training in hypoxia on sea-level performance: A meta-analysis. Sports Med. 2017;47:1651-60. https://doi. org/10.1007/s40279-017-0685-3

31. Chaudhary P, Suryakumar G, Prasad R, Singh SN, Ali S, Ilavazhagan G. Chronic hypobaric hypoxia mediated skeletal muscle atrophy: Role of ubiquitin-proteasome pathway and calpains. Mol Cell Biochem. 2012;364:101-13. https://doi.org/ 10.1007/s11010-011-1210-X

32. Vogt $\mathbf{M}$, Hoppeler $\mathbf{H}$. Is hypoxia training good for muscles and exercise performance? Prog Cardiovasc Dis. 2010;52:525-33. https://doi.org/10.1016/j.pcad.2010.02.013

33. Meeuwsen T, Hendriksen IJ, Holewijn M. Training-induced increases in sea-level performance are enhanced by acute intermittent hypobaric hypoxia. Eur J Appl Physiol. 2001;84:283-90. https://doi.org/10.1007/s004210000363

34. Howald H, Pette D, Simoneau JA, Uber A, Hoppeler H, Cerretelli P. Effect of chronic hypoxia on muscle enzyme activities. Int J Sports Med. 1990;11:S10-4. https://doi. org/10.1055/s-2007-1024847

35. Michalczyk M, Czuba M, Zydek G, Zajac A, Langfort J. Dietary recommendations for cyclists during altitude training. Nutrients. 2016;8:377. https://doi.org/10.3390/nu8060377

36. Hympanova L, Mori da Cunha M, Rynkevic R, Zundel M, Gallego MR, Vange J, et al. Physiologic musculofascial compliance following reinforcement with electrospun polycaprolactone-ureidopyrimidinone mesh in a rat model. J Mech Behav Biomed Mater. 2017;74:349-57. https://doi.org/10.1016/j.jmbbm.2017.06.032

37. Córdova A, Escanero JF, Giménez M. Magnesium distribution in rats after maximal exercise in air and under hypoxic conditions. Magnes Res. 1992;5:23-7. 